\title{
Fatores associados à segurança alimentar nos domicílios brasileiros em $2009^{*}$
}

\author{
Lorena Vieira Costa \\ Maria Micheliana da Costa Silva ${ }^{* * *}$ \\ Marcelo José Braga \\ Viviani Silva Lírio
}

\section{Resumo}

Este estudo pretende identificar os fatores associados à segurança alimentar nos domicílios brasileiros, por meio de um modelo de escolha binária (logit). Considera-se como medida desse fenômeno a ingestão calórica domiciliar, com base nos dados da Pesquisa de Orçamentos Familiares (POF/IBGE) de 2008-2009. Embora apenas 12,4\% dos domicílios ainda não apresentem um nível de segurança alimentar adequado, os resultados deste trabalho mostram que fatores como situação de pobreza e carência de itens básicos de infraestrutura e tamanho do domicílio contribuem para a incidência de segurança alimentar nesses domicílios. Além disso, residir em áreas rurais apresenta-se mais favorável à segurança alimentar. Ressalta-se a não existência de diferenças entre o consumo calórico domiciliar das regiões Nordeste e Sudeste, embora residir na região Sul ainda seja favorável para os domicílios estarem seguros quanto ao aspecto alimentar. Ademais, reforça-se a importância da educação, que contribui para o consumo alimentar.

Palavras-chave: Segurança alimentar; Ingestão calórica; Pesquisa de Orçamento Familiar; Logit; Brasil.

\section{Abstract \\ Factors associated with Brazilian food security inhouseholds in 2009}

This study aims to identify the factors associated with food security in households, using a binary choice model (logit). It is considered as a measure of this phenomenon household caloric intake, based on data from the Household Budget Survey (POF/IBGE) from 2008-2009. Although only $12.4 \%$ of households still do not have an adequate level of food security, the results of this study show that factors such as poverty and lack of basic infrastructure and household size contribute to its incidence in these households. Moreover, living in rural areas has become more favorable to food security. It is noteworthy that there is no difference between the caloric intake in the Northeast and Southeast, while residing in the South is still favorable for food security. Furthermore, it reinforces the importance of education, which contributes to food intake.

Keywords: Food security; Caloric intake; Household Budget Survey, Logit; Brazil.

JEL I31, C25, R20.

* Trabalho recebido em 21 de março de 2012 e aprovado em 8 de maio de 2013.

** Doutoranda em Economia Aplicada do Departamento de Economia Rural da Universidade Federal de Viçosa (UFV), Viçosa, MG, Brasil. E-mail: lorenavieiraufv@yahoo.com.br.

*** Doutoranda em Teoria Econômica da Universidade de São Paulo (USP), São Paulo, SP, Brasil. E-mail: maria.micheliana@ufv.br.

**** Professor-Associado IV do Departamento de Economia Rural da Universidade Federal de Viçosa (UFV), Viçosa, MG, Brasil. E-mail:mjbraga@ufv.br.

${ }^{* * * * *}$ Professora-Associada I do Departamento de Economia Rural da Universidade Federal de Viçosa (UFV), Viçosa, MG, Brasil. E-mail: viviani.lirio@gmail.com. 


\section{Introdução}

A extensão da fome e da insegurança alimentar é um importante indicador do padrão de vida de uma sociedade, como destacam Anand e Harris (1990). No Brasil, de acordo com Belik (2003), desde o início do século XX o tema da segurança alimentar passou a contar com políticas públicas mais evidentes, principalmente voltadas para a política agrícola, sistemas de abastecimento, controle de preços, distribuição de alimentos, entre outros. A partir de 1996, no entanto, essas intervenções pontuais do lado da produção e do consumo assumiram outra dimensão e ganharam novos objetivos com o compromisso assumido pelo Brasil e outra centena de países na Cúpula Mundial da Alimentação em Roma. O compromisso firmado entre os países refere-se à redução pela metade do número de pessoas atingidas pela desnutrição até o ano de 2015.

No entanto, como destaca Maluf (2006), as avaliações das metas acordadas na Cúpula são pessimistas quanto ao cumprimento daquele objetivo. Segundo dados da Organização das Nações Unidas para Agricultura e Alimentação - FAO (2010), entre os anos 1997 e 2009, houve uma tendência crescente do número de pessoas subnutridas no mundo. Em 2009, atingiu-se o pico de mais de 1 bilhão de pessoas subnutridas, correspondendo a $18 \%$ da população mundial, em parte devido à crise financeira global e aos altos preços de alimentos naquele ano. Embora esse número tenha se reduzido em 2010 para 16\%, os índices continuam acima do alvo estipulado pelo primeiro Objetivo do Milênio de reduzir a proporção de pessoas subnutridas para $10 \%$ até 2015 .

No caso brasileiro, os dados da Pesquisa Nacional por Amostra de Domicílios (PNAD) de 2004 e 2009 revelam que, entre esses anos, o percentual de domicílios em situação de insegurança alimentar caiu de 34,9\% em 2004, para $30,2 \%$, em 2009. Mesmo com a queda do percentual de domicílios em situação de insegurança alimentar, nota-se uma discrepância de sua incidência entre as regiões brasileiras. Enquanto no Sul, 18,7\% dos domicílios enfrentavam algum tipo de insegurança alimentar em 2009, no Nordeste e Norte, $46,1 \%$ e $40,2 \%$ dos domicílios, respectivamente, encontravam-se nessa situação. Ressalta-se, ainda, que a incidência de insegurança alimentar moderada e grave ${ }^{1}$ na região Nordeste é quase quatro vezes maior que na região Sul (21,3\% contra 5,4\% no Sul) (IBGE, 2010).

Além disso, segundo o Instituto Brasileiro de Análises Sociais e Econômicas (Ibase, 2008), entre as famílias assistidas pelo Programa Bolsa Família, 54,8\% estavam em situação de insegurança alimentar grave ou moderada,

(1) Conforme a Escala Brasileira de Insegurança Alimentar, que classifica a situação de segurança alimentar em quatro graus: segurança alimentar, insegurança alimentar leve, insegurança alimentar moderada e insegurança alimentar grave. Para mais detalhes, ver IBGE (2010). 
indicando que 6,1 milhões de famílias passaram por restrições na quantidade de alimentos ou por situações de fome nos três meses anteriores à pesquisa.

Dessa forma, o Brasil, além de ser um dos países mais desiguais quanto à distribuição de renda, como já é bem documentado pela literatura ${ }^{2}$, considerandose outras importantes dimensões relacionadas à desigualdade social, principalmente em relação ao acesso à alimentação, os resultados brasileiros indicam condições de vida inadequadas para o contingente da população mais pobre, como ressaltado no Relatório de Repercussão do Programa Bolsa Família (Ibidem).

A identificação dos fatores que colaboram para a segurança alimentar contribui para melhor focalização de políticas públicas que visam combater o estado de má nutrição persistente no país, uma vez que aponta as direções e a importância dos efeitos de variáveis socioeconômicas sobre esse fenômeno. Nesse contexto, o estudo pretende identificar fatores associados à segurança alimentar nos domicílios brasileiros. Especificamente, pretende-se analisar como os principais fatores relacionados ao acesso, à disponibilidade e à suficiência de alimentos nos domicílios concorrem para a ingestão de calorias pelo domicílio, e consequentemente, para seu estado de segurança alimentar.

Conforme Pérez-Escamilla e Segall-Corrêa (2008), há diferentes métodos que podem ser utilizados para avaliar a situação de segurança alimentar. As medidas podem ser agrupadas em objetivas e subjetivas. No primeiro caso, há os métodos baseados na antropometria ou aqueles que estabelecem um nível apropriado de consumo calórico ou de nutrientes que pode ser nacional, domiciliar ou individual. Essas medidas são, em grande parte, fundamentadas em pesquisas de renda e orçamentos familiares. As medidas subjetivas, por sua vez, são baseadas na percepção individual ou do responsável pelo domicílio quanto ao problema de insuficiência de renda familiar e alimentos. A literatura nacional tem-se baseado nesse último grupo de medidas, utilizando a Escala Brasileira de Insegurança Alimentar (EBIA), como em Hoffmann (2008), Camelo, Tavares e Saiani (2009) e Gubert, Benício e Santos (2010). Cabe destacar que embora a EBIA seja uma medida subjetiva, ela resulta em um algoritmo, dado por meio da classificação do domicílio entre os níveis de segurança alimentar, que pode ser analisado estatisticamente, como procedido por esses autores.

Contrariamente aos trabalhos supracitados, este trabalho utiliza a ingestão calórica domiciliar, fundamentada nos dados da Pesquisa de Orçamentos Familiares (POF/IBGE) de 2008-2009. Dessa forma, tem-se uma medida objetiva, baseada no real consumo alimentar das famílias, com o intuito de contemplar mais uma importante dimensão da segurança alimentar. Além disso, uma vez que as

(2) Como exemplos, há os trabalhos de Ferreira (2000); Paes de Barros; Henriques e Mendonça (2000) e Soares (2008) 
pesquisas baseadas em questionários subjetivos captam a situação alimentar relacionada a renda e situação de pobreza, a utilização de medidas objetivas permite a análise de segurança alimentar para outros níveis de renda. Como ressalta Maluf (2006), a segurança alimentar diz respeito ao conjunto da sociedade e não apenas aos seus segmentos de menores rendas.

De acordo com Babu e Sanyal (2009), embora a ingestão de alimentos inclua proteínas e outros nutrientes, a ingestão calórica é um dos principais parâmetros da segurança alimentar. Conforme os autores, o requerimento de calorias para um indivíduo é a quantidade de energia da dieta necessária para manter sua saúde, seu crescimento e um nível apropriado de atividade física. Para Smith (2002), a deficiência energética domiciliar está intimamente relacionada à noção de acesso aos alimentos e consiste numa boa medida de insegurança alimentar.

$\mathrm{O}$ artigo está organizado em quatro três, além desta introdução. A seguir apresenta-se uma revisão de literatura sobre a segurança alimentar e seus determinantes. A mensuração da segurança alimentar, bem como a identificação dos fatores associados a ela estão explícitos na segunda seção, que trata da metodologia. Os resultados obtidos e a discussão dos mesmos são apresentados na terceira seção e, por fim, vem a conclusão.

\section{Segurança alimentar e seus determinantes}

De acordo com a Lei Orgânica de Segurança Alimentar e Nutricional (Lei n. 11.346 de 15 de setembro de 2006),

a segurança alimentar e nutricional consiste na realização do direito de todos ao acesso regular e permanente a alimentos de qualidade, em quantidade suficiente, sem comprometer o acesso a outras necessidades essenciais, tendo como base práticas alimentares promotoras de saúde que respeitem a diversidade cultural e que sejam ambiental, cultural, econômica e socialmente sustentáveis (Brasil, 2006).

Ressalta-se nesse conceito a característica multidimensional do processo, que envolve toda a cadeia alimentar, sendo a segurança alimentar determinada por fatores que vão desde a produção agrícola, distribuição, possibilidade de acesso físico e econômico aos alimentos até a própria escolha alimentar, baseada em componentes culturais (Gubert; Benício; Santos, 2010). Assim, de acordo com Pérez-Escamilla e Segall-Corrêa (2008), a segurança alimentar de um domicílio, bem como a segurança nutricional individual, depende de fatores locais, regionais, nacionais e globais.

Ao se perseguir a segurança alimentar, objetiva-se, segundo Maluf (2006), combinar ações emergenciais, tais como assistenciais e compensatórias de combate 
à fome, visando assegurar tanto o acesso aos alimentos sem, contudo, comprometer parcela substancial da renda familiar, quanto a disponibilidade de alimentos de qualidade, originados de formas produtivas eficientes e sustentáveis. Além disso, de suma importância são as ações de divulgações de informações ao consumidor sobre práticas alimentares saudáveis e possíveis riscos à saúde, mediados pelo alimento.

Segundo a FAO (1996), a segurança alimentar existe quando todas as pessoas, em todos os momentos, têm acesso físico, social e econômico a alimentos suficientes, seguros e nutritivos que satisfaçam as suas necessidades dietéticas e preferências alimentares para uma vida ativa e saudável. Dessa forma, o conceito de segurança alimentar abrange três dimensões, frequentemente citadas ${ }^{3}$ : acesso, disponibilidade e suficiência na alimentação. Conforme Maxwell e Frankenberger (1992, apud Camelo; Tavares; Saiani, 2009), a disponibilidade de alimentos e o acesso a eles são fatores diretamente associados à renda familiar e às características do mercado de alimentos da região, como as possibilidades de produção, o sistemas de preço e de logística. A suficiência alimentar, por sua vez, depende da interação entre disponibilidade e acesso, além das decisões familiares de alocação do alimento disponível. Trata-se do modo pelo qual o alimento consumido é traduzido em benefícios nutricionais e para a saúde dos indivíduos (Babu; Sanyal, 2009).

Enquanto a disponibilidade de alimentos e as condições de infraestrutura, tais como estradas e escoamento, determinam o acesso físico ao alimento, o acesso econômico depende do poder de compra da família e do nível de preços de alimentos (Thomson; Metz, 1998). Assim, Tweeten (1999) destaca que diante da grande possibilidade de que os alimentos estejam disponíveis nos mercados mundiais para qualquer indivíduo com poder de compra, provavelmente a acessibilidade seja uma das maiores restrições à segurança alimentar.

De acordo com Timmer, Falcon e Pearson (1983), o conhecimento dos fatores que afetam o padrão de consumo de alimentos da população é de suma importância para o estabelecimento de programas e políticas públicas de enfrentamento dos problemas alimentares da sociedade. Assim, deve-se entender de que forma a população altera seu padrão de consumo de alimentos diante de alterações na renda, nos preços de alimentos em relação aos preços de outros bens, mudanças no tamanho da família, do lugar da residência, entre outros fatores.

Hoffmann (2008), ao analisar os dados da PNAD 2004 avaliando os fatores determinantes da insegurança alimentar, medida pela Escala Brasileira de Insegurança Alimentar (EBIA), conclui que a baixa renda domiciliar per capita constitui o determinante isolado mais importante da insegurança alimentar. Além disso, a residência rural, atividade agrícola e a presença de pessoas com menos de

(3) Por exemplo: Babu e Sanyal (2009); Camelo; Tavares e Saiani (2009); Tweeten (1999), entre outros. 
18 anos no domicílio contribuem para reduzir a probabilidade de insegurança alimentar, assim como a maior escolaridade da pessoa de referência do domicílio. A probabilidade de insegurança alimentar se eleva quando a pessoa de referência é preta ou parda, tem ocupação instável e/ou informal. O autor constata ainda que a existência de luz elétrica, água encanada e esgoto apropriado reduzem a probabilidade de insegurança alimentar. Além disso, o fato de a pessoa de referência do domicílio ser mulher eleva a probabilidade de insegurança alimentar, mesmo que o valor médio da renda per capita desse domicílio seja mais alto do que quando a pessoa de referência é homem. O autor indaga se esse resultado se manteria caso se utilizasse uma medida de insegurança alimentar mais objetiva, assumindo a necessidade de maiores pesquisas sobre o tema.

Levando em consideração a importância da renda para a garantia de segurança alimentar, Camelo, Tavares e Saiani (2009) analisaram o impacto do Programa Bolsa Família (PBF) sobre a segurança alimentar dos domicílios beneficiários e verificaram que o PBF de fato eleva a probabilidade de os domicílios estarem em situação de segurança alimentar, medida pela Escala Brasileira de Insegurança Alimentar. No entanto, os autores verificaram que o programa afeta de forma significativa apenas os domicílios em situação de insegurança alimentar leve, não apresentando resultados expressivos sobre os beneficiários em condições mais severas. Diante disso, nota-se que embora a renda seja importante determinante da situação de insegurança alimentar de um domicílio, outros fatores subjacentes às dimensões da disponibilidade, acesso e suficiência contribuem de forma significativa.

A análise de identificação dos fatores associados à segurança alimentar não implica determinar a causalidade entre as variáveis analisadas e o fenômeno em questão. Conforme destacam Haughton e Khandker (2009), uma associação estatística sozinha não é suficiente para estabelecer causalidade. Para esse propósito, informações adicionais devem ser requeridas. Além disso, os autores ressaltam que em estudos sobre pobreza pode ser difícil separar a causalidade da correlação.

\section{Metodologia}

\subsection{Mensuração da segurança alimentar}

Diante da característica multidimensional do problema da insegurança alimentar, um dos desafios das pesquisas sobre o tema diz respeito à escolha do método de mensuração da mesma. Segundo Dutta e Gundersen (2007), nos últimos anos tem havido grande esforço no sentido de se utilizarem medidas diretas de insegurança alimentar, tais como aquelas baseadas em pesquisas de consumo domiciliar de alimentos e medidas qualitativas, em contraste com métodos que se 
valem da análise de variáveis geralmente consideradas correlacionadas com a insegurança alimentar, como renda domiciliar per capita e medidas antropométricas.

Como ressaltam Babu e Sanyal (2009), deve-se considerar que diferentes indivíduos possuem diferentes necessidades calóricas. Nesse sentido, segundo os autores, é necessária a utilização de um sistema de pesos, tais como escalas de equivalência. Para um domicílio de um determinado tamanho e composição demográfica, uma escala de equivalência mensura o número de homens adultos equivalentes que aquele domicílio possui. Assim, cada membro do domicílio é contado como alguma fração de um homem adulto. Desse modo, o tamanho do domicílio é a soma dessas frações e é medido em números de adultos equivalentes e não em números de pessoas. Um modo comum de mensurar essa equivalência, citada pelos autores e adotado neste trabalho, é a escala da Organização para a Cooperação e Desenvolvimento Econômico - OECD - de adultos equivalentes (AE), que pode ser escrita como:

$$
A E=1+0,7\left(N_{\text {adultos }}-1\right)+0,5\left(N_{\text {crianças }}\right),
$$

Assim, um domicílio que contenha apenas um adulto terá a equivalência em adulto de 1, um domicílio com dois adultos terá uma equivalência de 1,7. Nesse sentido, 0,7 representa economias de escala sobre o acréscimo de mais um adulto no domicílio e 0,5 refere-se ao peso dado às crianças, uma vez que se presume que elas tenham menores necessidades.

A medida de segurança alimentar considerada neste trabalho é baseada na ingestão calórica utilizando a equivalência em adultos para cada domicílio. Assim, a segurança alimentar é definida como a capacidade do domicílio de satisfazer ao menos $80 \%$ da ingestão calórica individual que é requerida, ou seja, em média $2.500 \mathrm{kcal}$ diárias, quantidade sugerida pela Agência Nacional de Vigilância Sanitária (Anvisa, 2001).

\subsection{Identificação dos fatores associados à segurança alimentar}

De acordo com Camelo, Tavares e Saiani (2009), as variáveis que explicam a segurança alimentar devem estar relacionadas às três dimensões que a conceituam: acesso, disponibilidade e suficiência. Dessa forma, para verificar se o j-ésimo domicílio está seguro, tem-se:

$$
y_{j}=G(A, D, S)=G(\alpha+A \beta+D \gamma+S \delta+\varepsilon),
$$

em que: A é um vetor de variáveis relacionadas ao acesso; D é um vetor de variáveis relacionadas à disponibilidade e $\mathrm{S}$ é um vetor com variáveis que expressam a suficiência; $\alpha, \beta, \gamma, \delta$ são os parâmetros relacionados a cada vetor de 
variáveis e $\varepsilon$ pode ser considerado como os fatores não observados, relacionados às preferências alimentares.

A análise da segurança alimentar baseada na ingestão calórica alimentar de um domicílio sugere a elaboração de um problema de escolha binária. Isso porque, como visto, para o domicílio estar seguro, em média ${ }^{4}$, deverá atingir pelo menos $80 \%$ do que é requerido em calorias. Dessa forma, a variável de interesse tem a seguinte definição:

$$
y_{j}=1 \text {, caso o j-ésimo domicílio tenha atingido a quantidade mínima de }
$$

calorias requeridas;

$$
y_{j}=0 \text {, caso contrário. }
$$

Como a probabilidade de ocorrência de $y_{j}$ pode ser expressa como $p\left(y_{j}\right)$, expressa-se o modelo como (WOOLDRIDGE, 2008):

$$
p\left(y_{j}=1 \mid A, D, S\right)=G(\alpha+A \beta+D \gamma+S \delta+\varepsilon),
$$

ou seja, a variável dependente do modelo é uma variável limitada. Nesse caso, a estimação do modelo por Mínimos Quadrados Ordinários (MQO) apresenta alguns problemas $^{5}$. Assim, recorre-se ao modelo logit, que utiliza uma função de distribuição logística acumulada:

$$
p\left(y_{j}\right),=G(\alpha+A \beta+D \gamma+S \delta+\varepsilon)=G(z)=\frac{\exp (z)}{1+\exp (z)}=\Lambda(z),
$$

Já a probabilidade de não ocorrência é expressa por $1-p\left(y_{j}\right)$, desta forma:

$$
1-p\left(y_{j}\right)=\frac{1}{1+\exp (z)}
$$

Assim, as chances favoráveis para a ocorrência do evento, nesse caso, de o domicílio estar seguro, são dadas por:

$$
\frac{p\left(y_{j}\right)}{1-p\left(y_{j}\right)}=\exp (z)
$$

Como (6) é não linear nos parâmetros, deve ser estimada na sua forma linearizada:

$$
L=\ln \left(\frac{p\left(y_{j}\right)}{1-p\left(y_{j}\right)}\right)=G(\alpha+A \beta+D \gamma+S \delta+\varepsilon),
$$

Dessa forma, pode-se verificar a contribuição de cada fator na probabilidade de o i-ésimo domicílio estar em uma situação de segurança alimentar.

(4) Considerando a ponderação do adulto equivalente.

(5) Ver Wooldridge (2008). 


\subsection{Variáveis e fontes dos dados}

De acordo com as três dimensões, e com base nos trabalhos de Hoffmann (2008) e Camelo, Tavares e Saiani (2009), este trabalho considera como variáveis determinantes da segurança alimentar:

i. Acesso: Variáveis relacionadas aos rendimentos e condições do domicílio Renda domiciliar per capita, baseada no salário mínimo de 2009 (ano da pesquisa, cujo valor equivale a $\mathrm{R} \$ 465,00$ ), infraestrutura, características do chefe de domicílio (idade, sexo) e dos demais moradores (escolaridade média), participação de aposentadorias e pensões na renda familiar;

ii. Disponibilidade: Variáveis relacionadas à localização, como proxies de disponibilidade de alimentos, devido às características regionais;

iii. Suficiência: Variáveis relacionadas ao tamanho da família (número de crianças e de idosos, densidade morador/cômodo) e alocação de recursos para a alimentação dentro do domicílio (proporção dos gastos com alimentos dentro do domicílio sobre a renda total domiciliar).

Em vez de usar a escolaridade do chefe de domicílio, como em Hoffmann (2008), considera-se a escolaridade média do domicílio, visto que as decisões individuais podem diferenciar o consumo alimentar entre indivíduos de um mesmo domicílio. Além disso, este trabalho considera a participação dos gastos com alimentação dentro do domicílio em relação à renda domiciliar total, para captar a suficiência da alimentação domiciliar. Vale ressaltar que a utilização de variáveis indicativas da região em que o domicílio está localizado como uma das proxies da disponibilidade de alimentos possui limitações. Uma delas diz respeito ao fato de que determinadas regiões possuem um conjunto de fatores que podem melhorar a situação das pessoas quanto à alimentação, como uma melhor infraestrutura e escolaridade e rendas médias maiores. No entanto, estar em determinada região também pode ser fator que facilita ou dificulta a disponibilidade de alimentos. Desse modo, a localização pode refletir tanto o acesso aos alimentos quanto a disponibilidade dos mesmos.

Todas essas variáveis, assim como a ingestão calórica utilizada para a medida de segurança alimentar, foram extraídas da base de dados da Pesquisa de Orçamentos Familiares (POF), realizada pelo IBGE para os anos 2008-2009.

Foram considerados os domicílios que continham todas as informações relevantes à análise, excluindo as observações que continham missing value ou valores discrepantes.

De acordo com os grupos de variáveis explicativas do modelo, relacionadas ao acesso aos alimentos e a sua disponibilidade e suficiência nos domicílios, a Tabela 1 apresenta as respectivas estatísticas descritivas para cada variável. A maioria dos domicílios (cerca de 40\%) está situada no estrato 
intermediário, que possui renda per capita entre um e três salários mínimos de 2009. No primeiro estrato, que considera os domicílios em situação de pobreza, encontram-se $20,3 \%$ dos domicílios. Apenas $6 \%$ da amostra está no estrato de renda mais alto. Em média, a participação de aposentadorias e pensões sobre o rendimento domiciliar equivale a $21,9 \%$. A idade média dos chefes de domicílio é 49 anos, sendo que 69\% são do sexo masculino. Praticamente todos os domicílios têm fogão, 93\% possuem geladeira e 91\% possuem água canalizada, embora apenas $35,7 \%$ tenham acesso à rede de esgoto.

Tabela 1

Estatísticas descritivas das variáveis explicativas

\begin{tabular}{|c|c|c|}
\hline Variáveis & & Média \\
\hline \multicolumn{3}{|l|}{ Acesso } \\
\hline \multirow[t]{6}{*}{$\mathrm{RDPC} *$} & {$[0 ; 0.5]$} & $20,29 \%$ \\
\hline & $(0.5 ; 1]$ & $26,13 \%$ \\
\hline & $(1 ; 3]$ & $39,63 \%$ \\
\hline & $(3 ; 5]$ & $7,77 \%$ \\
\hline & $>5$ & $6,17 \%$ \\
\hline & $\%$ aposentadorias e pensões na renda & $21,92 \%$ \\
\hline \multirow{2}{*}{ Chefe do domicílio } & Idade & 49 \\
\hline & $\%$ de chefes do sexo masculino & $69,36 \%$ \\
\hline \multirow[t]{5}{*}{ Infraestrutura } & \% de domicílios com fogão & $99,23 \%$ \\
\hline & $\%$ de domicílios com geladeira & $92,77 \%$ \\
\hline & \% de domicílios com água canalizada & $90,83 \%$ \\
\hline & $\%$ de domicílios com rede de esgoto & $35,70 \%$ \\
\hline & Escolaridade média do domicílio & 6,04 \\
\hline \multicolumn{3}{|l|}{ Disponibilidade local } \\
\hline \multirow[t]{6}{*}{ Região } & $\%$ de domicílios situados na zona urbana & $78,19 \%$ \\
\hline & $\%$ de domicílios situados no Norte & $14,09 \%$ \\
\hline & $\%$ de domicílios situados no Nordeste & $36,24 \%$ \\
\hline & $\%$ de domicílios situados no Sudeste & $23,08 \%$ \\
\hline & $\%$ de domicílios situados no Centro-Oeste & $13,33 \%$ \\
\hline & $\%$ de domicílios situados no Sul & $13,26 \%$ \\
\hline \multicolumn{3}{|l|}{ Suficiência } \\
\hline & N. de crianças & 1,1328 \\
\hline & N. de idosos & 0,4710 \\
\hline & Densidade morador/cômodo & 0,6459 \\
\hline & $\begin{array}{l}\text { \% despesa com alimentação dentro do } \\
\text { domicílio em relação à renda }\end{array}$ & $5,08 \%$ \\
\hline N. de observações & & 13.842 \\
\hline
\end{tabular}

(*) Renda domiciliar per capita, referente ao salário mínimo de 2009 (R\$ 465,00). Fonte: Elaboração própria, a partir das informações da POF 2008-2009. 
A maioria dos domicílios está localizada na área urbana (78\%) e na região Nordeste $(36 \%)$. Em média, os domicílios têm uma criança e 0,6 morador por cômodo. A participação dos gastos com alimentação dentro do domicílio em relação à renda total equivale a 5\%, em média.

A amostra considera 13.842 domicílios $^{6}$, representando 14.309 .709 domicílios brasileiros, quando aplicado o fator de expansão da POF/IBGE.

\section{Resultados e discussão}

A partir da amostra analisada, o número de domicílios que apresentaram ingestão calórica abaixo do necessário ( $80 \%$ de 2.500 calorias) foi de 1.711 domicílios, o que correspondeu a $12,4 \%$ do total de domicílios analisados. Esse resultado mostra que a insegurança alimentar, quando medida por uma de suas dimensões - ou seja, a insuficiência calórica alimentar - é substancialmente menor do que aquela encontrada por meio da EBIA, baseada na percepção individual quanto ao problema, que foi de 30,2\% em 2009 (IBGE, 2010).

Ao considerar as faixas de renda per capita $(\mathrm{RDPC})^{7}$, nota-se a discrepância em relação às condições de renda e ao nível de insegurança alimentar dos domicílios, como se verifica na Tabela 2.

Tabela 2

Domicílios em situação de insegurança alimentar de acordo com os estratos de renda per capita, Brasil, 2008-2009

\begin{tabular}{cccc}
\hline Estratos de RDPC & N. de domicílios & $\begin{array}{c}\text { N. de domicílios com } \\
\text { insegurança alimentar }\end{array}$ & $\%$ \\
\hline$[0 ; 0.5]$ & 2.809 & 590 & 21,0 \\
$(0.5 ; 1]$ & 3.617 & 493 & 13,6 \\
$(1 ; 3]$ & 5.486 & 479 & 8,7 \\
$(3 ; 5]$ & 1.076 & 85 & 7,9 \\
$>5$ & 854 & 64 & 7,5 \\
Total & 13.842 & 1.711 & 12,4 \\
\hline
\end{tabular}

Fonte: Resultados da pesquisa.

Como observado, a maioria dos domicílios que apresentam consumo alimentar insuficiente em termos calóricos possui renda domiciliar per capita inferior a meio salário mínimo, ou seja, está em situação de pobreza. Entretanto, mesmo em níveis de renda mais alto, a situação de insegurança alimentar ainda é

(6) Já desconsiderados os domicílios que não apresentaram nenhuma informação sobre o consumo calórico ou com ausência de informações importantes.

(7) Estratos de renda baseados no salário mínimo de 2009 (R\$ 465,00). 
persistente, evidenciando que outros fatores, além da renda, contribuem para tal situação.

Segundo Hoffmann (2008), o nível de renda domiciliar per capita é o fator mais importante para explicar a situação de insegurança alimentar. Entretanto, segundo o autor, a localização do domicílio também pode contribuir significativamente para o fenômeno, uma vez que ela reflete a disponibilidade de alimentos. Dessa forma, a Tabela 3 expõe a proporção de domicílios em estado de insegurança alimentar por zona de residência e região geográfica.

Tabela 3

Insegurança alimentar de acordo a zona de residência e região geográfica, Brasil, 2008-2009

\begin{tabular}{ccccc}
\hline \multirow{2}{*}{ Localização } & & $\begin{array}{c}\text { N. de } \\
\text { domicílios }\end{array}$ & $\begin{array}{c}\text { N. de domicílios } \\
\text { com insegurança } \\
\text { alimentar }\end{array}$ & $\%$ \\
\hline \multirow{2}{*}{ Zona de residência } & Urbana & 10.823 & 1.331 & 12,3 \\
& Rural & 3.019 & 380 & 12,6 \\
\hline \multirow{2}{*}{ Regiões } & Norte & 1.951 & 269 & 13,8 \\
& Nordeste & 5.016 & 712 & 14,2 \\
& Centro-Oeste & 1.845 & 261 & 14,2 \\
& Sudeste & 3.195 & 345 & 10,8 \\
& Sul & 1.835 & 124 & 6,8 \\
\hline
\end{tabular}

Fonte: Resultados da pesquisa.

Nota-se que os domicílios urbanos e rurais apresentam incidência similar de insegurança alimentar, dada pela ingestão calórica. No entanto, ao considerar a dimensão subjetiva e perceptiva desse fenômeno, dada pela EBIA, a insegurança alimentar foi maior nas áreas rurais, de acordo com Hoffmann (2008) para o ano de 2004. Embora este trabalho e o de Hoffmann (2008) não possam ser diretamente comparados, uma vez que se trata de fontes de dados e métodos de medidas de insegurança alimentar diferentes, a observação dos diferentes efeitos encontrados é importante na medida em que possibilitam evidenciar os fatores que se relacionam ao fenômeno em estudo de forma multidimensional - tanto quando o mesmo é medido com base na ingestão calórica, quanto quando se tem uma medida baseada na percepção individual.

Verifica-se que a região brasileira que apresenta menor nível de insegurança alimentar é a região Sul (6,8\%), seguida da região Sudeste $(10,8 \%)$. No Nordeste e no Centro-Oeste, que apresentam ocorrência semelhante de segurança alimentar, estão os maiores níveis: $14,2 \%$. Resultado semelhante foi encontrado por Gubert, Benício e Santos (2010) ao utilizarem a EBIA. Esses autores notaram que a região com maior ocorrência de insegurança alimentar foi a 
região Nordeste e a de menor prevalência, a região Sul. Para verificar o impacto dos fatores relacionados ao acesso aos alimentos e à sua disponibilidade e suficiência sobre a probabilidade de os domicílios estarem seguros, a Tabela 4 mostra os resultados do modelo logit com erros padrão robustos e as razões de chances favoráveis à situação de segurança alimentar.

Pela estatística de Wald verifica-se que as variáveis utilizadas são conjuntamente significativas, com probabilidade caudal de nulidade conjunta dos parâmetros igual a $0 \%$. Os coeficientes apresentados na Tabela 4 apenas representam o logaritmo das razões de chances favoráveis. Entretanto, o sinal de cada coeficiente indica a direção do impacto de cada variável sobre a segurança alimentar.

Os coeficientes que indicam a proporção de rendimentos provenientes de aposentadorias e pensões, o diferencial de residir na região Centro-Oeste e na zona urbana, a quantidade de crianças na faixa de zero a 6 anos e a densidade morador/cômodo apresentaram sinal negativo. O coeficiente diferencial para as regiões Norte e Sudeste, bem como a existência de rede de esgotamento sanitário são estatisticamente insignificantes para explicar a segurança alimentar, em comparação às suas respectivas bases. Dessa forma, não há diferença significativa entre a região Nordeste e as regiões Sudeste e Norte em relação à ingestão calórica domiciliar. O mesmo pode-se concluir sobre a probabilidade de se estar nessa situação entre domicílios com e sem rede de esgotamento sanitário. A idade do chefe de domicílio contribui negativamente para o domicílio estar seguro, no entanto, a partir da idade aproximada de $45 \operatorname{anos}^{8}$, a contribuição passa a ser positiva. As demais variáveis apresentaram impactos positivos e significativos.

Tabela 4

Resultados do modelo logit e razão de chances favoráveis para a segurança alimentar, Brasil, 2008-2009

\begin{tabular}{llrrrc}
\hline \multirow{2}{*}{ Variável } & & Coeficiente & $\begin{array}{c}\text { Desvio } \\
\text { padrão }\end{array}$ & $\begin{array}{c}\text { Razão } \\
\text { de } \\
\text { chances }\end{array}$ \\
\hline Acesso & Constante** & 0,6991 & 0,3666 & - \\
& $(0,5 ; 1]^{* * *}$ & 0,3743 & 0,0800 & 1,4539 \\
RDPC & $(1 ; 3]^{* * *}$ & 0,7943 & 0,0964 & 2,2129 \\
& $(3 ; 5]^{* * *}$ & 0,8430 & 0,1533 & 2,3234 \\
& $>5^{* * *}$ & 0,8120 & 0,1761 & 2,2524 \\
& $\%$ aposentadorias e pensões na renda $* *$ & $-0,2745$ & 0,1106 & 0,7599 \\
\hline & & & & & \multicolumn{2}{c}{ Continua }
\end{tabular}

(8) Esse valor é encontrado pela derivação do efeito marginal com respeito à variável idade e a seu valor quadrático, considerando a derivada no ponto médio, ou seja, na idade média dos chefes de domicílio. 
Tabela 4 - Continuação

\begin{tabular}{|c|c|c|c|c|}
\hline Variável & & Coeficiente & $\begin{array}{l}\text { Desvio } \\
\text { padrão }\end{array}$ & $\begin{array}{l}\text { Razão } \\
\text { de } \\
\text { chances }\end{array}$ \\
\hline \multirow{4}{*}{$\begin{array}{l}\text { Chefe do } \\
\text { domicílio }\end{array}$} & idade** & $-0,0211$ & 0,0104 & 0,9791 \\
\hline & idade $2 * *$ & 0,0002 & 0,0001 & 1,0002 \\
\hline & sexo masculino $* * *$ & 0,2542 & 0,0588 & 1,2894 \\
\hline & fogão** & 0,4528 & 0,2353 & 1,5728 \\
\hline \multirow{2}{*}{ Infraestrutura } & geladeira $* * *$ & 0,2708 & 0,0945 & 1,3110 \\
\hline & água canalizada* & 0,1703 & 0,0954 & 1,1857 \\
\hline & rede de esgoto & 0,0023 & 0,0682 & 1,0023 \\
\hline & escolaridade média do domicílio*** & 0,0333 & 0,0111 & 1,0338 \\
\hline \multicolumn{5}{|l|}{ Disponibilidade local } \\
\hline & zona urbana** & $-0,1446$ & 0,0730 & 0,8653 \\
\hline & Norte & 0,0922 & 0,0849 & 1,0965 \\
\hline \multirow{3}{*}{ Região } & Sudeste & 0,0483 & 0,0801 & 1,0495 \\
\hline & Centro-Oeste** & $-0,2035$ & 0,0830 & 0,8159 \\
\hline & $\mathrm{Sul}^{* * *}$ & 0,4697 & 0,1066 & 1,5994 \\
\hline \multicolumn{5}{|l|}{ Suficiência } \\
\hline & $0-6$ anos $* * *$ & $-0,3536$ & 0,0403 & 0,7021 \\
\hline \multirow[t]{5}{*}{ N. de crianças } & $7-15$ anos $* * *$ & 0,1301 & 0,0344 & 1,1389 \\
\hline & $16-18$ anos*** & 0,4743 & 0,0760 & 1,6069 \\
\hline & n. de idosos $* *$ & 0,1693 & 0,0687 & 1,1845 \\
\hline & densidade morador/cômodo $* * *$ & $-0,1468$ & 0,0599 & 0,8635 \\
\hline & $\begin{array}{l}\text { \% despesa com alimentação dentro do } \\
\text { domicílio em relação à renda*** }\end{array}$ & 3,0274 & 1,0322 & 20,6438 \\
\hline N. de observações & & & & 13842 \\
\hline Wald $\chi^{2}(24)$ & & & & 593,65 \\
\hline Prob $>\chi^{2}$ & & & & 0,0000 \\
\hline Classificação correta & & & & $87,6 \%$ \\
\hline
\end{tabular}

Nível de significância: $* 10 \%, * * 5 \%, * * * 1 \%$.

Grupo-base: estrato de RDPC [0;0.5], região Nordeste, chefe de domicílio do sexo masculino, zona urbana e presença dos itens de infraestrutura.

Fonte: Resultados da pesquisa.

Camelo, Tavares e Saiani (2009), analisando a contribuição do Programa Bolsa Família por meio da EBIA, também encontraram sinais semelhantes em relação à idade do chefe de família, à área urbana, à densidade morador/cômodo e ao número de crianças de zero a seis anos, embora este último não tenha sido significativo para o trabalho dos autores. 
O sinal encontrado pelas estimações expostas na Tabela 4 é favorável ao que se espera sobre o impacto de se ter acesso à água canalizada, uma vez que ter acesso a água, principalmente oriunda de redes de tratamento, tem importante contribuição tanto para a utilização adequada de alimentos, quanto para a produção de subsistência.

$\mathrm{Na}$ última coluna da Tabela 4, apresentam-se as razões de chance de ocorrência da segurança alimentar nos domicílios, já transformadas da sua forma logarítmica. No primeiro grupo, que representa as variáveis correspondentes ao acesso a alimentos, nota-se que as chances de os domicílios estarem em situação de segurança alimentar aumentam com os estratos de renda per capita, sendo que para o último estrato as chances de estar em um nível de segurança alimentar adequado são 2,25 vezes maiores do que para os domicílios com renda per capita de até meio salário mínimo. No entanto, é o $4^{\circ}$ estrato que tem maior chance favorável. Em relação à composição da renda familiar, quanto mais importante é a renda proveniente de aposentadorias e pensões, menores as chances favoráveis de esse domicílio estar seguro (equivalente a $3 / 4$ de chance).

Caso o chefe do domicílio seja do sexo masculino, tem-se 1,3 vez mais chances. Esse resultado é semelhante ao de Schlindwein e Kassouf (2007), que analisaram as mudanças no padrão de consumo das famílias brasileiras. No estudo, as autoras observam que é cada vez mais comum a inserção de mulheres no mercado de trabalho, o que as obriga a dividir seu tempo entre gerar renda, cuidar da casa e dos filhos, tornando-as cada vez menos responsáveis diretamente pela alimentação familiar.

Para as variáveis relacionadas a condições de infraestrutura, que também podem refletir o acesso e a utilização dos alimentos, o fato de possuir um fogão ou geladeira elevam em 1,5 e 1,3 vezes as chances de estar seguro, respectivamente. É importante ressaltar a forte relação existente entre a posse da geladeira e a presença de luz elétrica no domicílio. Assim, nota-se que domicílios que possuem geladeira, e consequentemente energia elétrica, têm maiores chances de estar seguros. Com esse resultado, mostra-se a importância desse item, uma vez que é imprescindível para melhor armazenamento dos alimentos. A presença de água canalizada também eleva essas chances, em cerca de 1,2 vez. Hoffmann (2008) também verifica que a existência de luz elétrica e água encanada reduz a probabilidade de insegurança alimentar do domicílio e destaca que a influência dessas variáveis que representam a infraestrutura mostra que a probabilidade de insegurança alimentar para um domicílio depende do acesso a serviços que são, em grande parte, dependentes da atuação do Estado, como saneamento, fornecimento de água tratada, entre outros.

Além desses fatores, a escolaridade média do domicílio também eleva a probabilidade de o mesmo estar seguro em 1,03 vez, evidenciando o impacto do maior nível de escolaridade sobre padrão de consumo domiciliar. Hoffmann (2008), embora considerando a escolaridade da pessoa de referência do domicílio, 
também verifica a contribuição entre o nível de escolaridade e a situação favorável ao estado de segurança alimentar.

Em relação ao grupo de variáveis que reflete a disponibilidade de alimentos, verifica-se que residir em zona urbana apenas eleva em $0,86 \mathrm{vez}$ as chances de o domicílio estar em situação favorável à segurança alimentar, comparando-se à zona rural. Dessa forma, residir em zona urbana é menos favorável para garantir um consumo calórico suficiente. Esse fato pode ser explicado pela importância da produção de subsistência para os pequenos produtores rurais, o que garante o autoconsumo alimentar. Também Hoffmann (2008), ao controlar os efeitos da renda domiciliar per capita e da escolaridade, verifica que a residência rural e a atividade agrícola contribuem para reduzir a insegurança alimentar. Conforme o autor, pessoas com domicílio rural e ocupação agrícola possuem maior disponibilidade de alimentos.

Como já ressaltado, da comparação da região Nordeste (base) com as demais regiões, notou-se que não há diferenças significativas entre essa região e as regiões Norte e Sudeste. Contudo, residir na região Centro-Oeste reduz as chances de o domicílio estar em situação favorável à segurança alimentar, ao passo que residir na região Sul a aumenta mais de 1,5 vez.

Quanto às variáveis que dizem respeito à suficiência de alimentos, observa-se que um maior número de crianças de zero a seis anos reduz as chances de segurança alimentar do domicílio (apresentando chances favoráveis de 0,70). A partir de 7 anos, no entanto, o aumento do número de crianças contribui para elevação da probabilidade de segurança alimentar. Quanto à presença de idosos no domicílio, verifica-se que quanto maior o número de idosos, maior a probabilidade de o domicílio estar seguro (as chances favoráveis são de 1,18 vez). É possível notar, ainda, que quanto mais moradores por cômodo no domicílio, menor a probabilidade de o domicílio estar em estado de segurança alimentar, sendo que as chances favoráveis são de apenas 0,86 vez. Entretanto, quanto maior a proporção da renda alocada para a alimentação dentro do domicílio, maior a probabilidade de se estar seguro: as chances aumentam 20 vezes com a elevação dessa proporção.

Em média, a probabilidade estimada de um domicílio estar em situação de segurança alimentar é de $87,6 \%$. A menor probabilidade equivale a $23 \%$ enquanto a maior corresponde a $99 \%$. Na Tabela 5, observa-se o efeito marginal de cada variável sobre a probabilidade de estar em situação de segurança alimentar um domicílio que tenha as seguintes características: estar situado na área urbana nordestina; ter renda per capita domiciliar entre zero e meio salário mínimo; o chefe de domicílio ser do sexo feminino e não possuir nenhum item referente à infraestrutura (água canalizada, rede de esgoto, fogão e geladeira), que se apresentaram desfavoráveis. Mesmo com essas características não favoráveis, um domicílio com estas características apresenta a probabilidade de possuir ingestão calórica suficiente de $61,3 \%$. 
Tabela 5

Efeitos marginais das variáveis explicativas sobre a probabilidade de um domicílio estar em segurança alimentar, Brasil, 2008-2009

\begin{tabular}{|c|c|c|}
\hline Variáveis & & $\begin{array}{c}\text { Efeito } \\
\text { marginal }\end{array}$ \\
\hline \multicolumn{3}{|l|}{ Acesso } \\
\hline \multirow{6}{*}{ RDPC } & $(0,5 ; 1]$ & 0,0801 \\
\hline & $(1 ; 3]$ & 0,1551 \\
\hline & $(3 ; 5]$ & 0,1628 \\
\hline & $>5$ & 0,1579 \\
\hline & $\%$ aposentadorias e pensões na renda & $-0,0627$ \\
\hline & Idade * & 0,0005 \\
\hline \multirow{2}{*}{$\begin{array}{l}\text { Chefe do } \\
\text { domicílio }\end{array}$} & Sexo & 0,0557 \\
\hline & Fogão & 0,0954 \\
\hline \multirow{4}{*}{ Infraestrutura } & Geladeira & 0,0591 \\
\hline & Água canalizada & 0,0378 \\
\hline & Rede de esgoto & 0,0005 \\
\hline & Escolaridade média do domicílio & 0,0076 \\
\hline \multicolumn{3}{|c|}{ Disponibilidade local } \\
\hline & Zona urbana & $-0,0337$ \\
\hline & Norte & 0,0207 \\
\hline \multirow{3}{*}{ Região } & Sudeste & 0,0110 \\
\hline & Centro-Oeste & $-0,0477$ \\
\hline & Sul & 0,0986 \\
\hline \multicolumn{3}{|l|}{ Suficiência } \\
\hline & $0-6$ anos & $-0,0807$ \\
\hline \multirow[t]{5}{*}{ N. de crianças } & $7-15$ anos & 0,0297 \\
\hline & $16-18$ anos & 0,1083 \\
\hline & n. de idosos & 0,0387 \\
\hline & Densidade morador/cômodo & $-0,0335$ \\
\hline & $\begin{array}{l}\% \text { despesa com alimentação dentro do domicílio } \\
\text { na renda }\end{array}$ & 0,6912 \\
\hline Probabilidade & & $61,3 \%$ \\
\hline
\end{tabular}

* Para a variável idade, o efeito marginal é calculado da seguinte forma:

$$
\frac{d y}{d x}=\beta+2 \gamma \bar{x}
$$

em que: $\overline{\mathrm{x}}$ é a idade média; $\beta$ e $\gamma$ são os coeficientes do efeito marginal de idade e seu termo quadrático, respectivamente.

Fonte: Resultados da pesquisa. 
Dentre as variáveis referentes ao acesso de alimentos, a que apresentou efeito marginal maior foi o estrato de renda per capita de 3 a 5 salários mínimos. Nesse caso, a probabilidade de estar em situação de segurança alimentar aumenta 16,3 pontos percentuais (p.p.), em comparação com o $1^{\circ}$ estrato de RDPC. Ao passar do $1^{\circ}$ para o $2^{\circ}$ estrato, a probabilidade eleva-se em apenas 8 p.p. Já para o último, o acréscimo é de 15,8 p.p. Embora seja o estrato de renda mais alto, a sua contribuição é menor do que a do $4^{\circ}$ estrato. Em relação à composição dos rendimentos domiciliares, o aumento de 1 p.p. da participação de aposentadorias e pensões na renda reduz a probabilidade de segurança alimentar em 6,3 p.p.

As variáveis relacionadas ao chefe de domicílio apresentaram baixa contribuição, principalmente a idade, cujo incremento é de 0,05 p.p. A existência de fogão no domicílio apresenta a maior contribuição nesse grupo, depois das variáveis referentes às faixas de rendimento, sendo seu efeito marginal equivalente a cerca de 10 p.p. A contribuição da escolaridade média do domicílio é de apenas 0,76 p.p.

Verificando as variáveis de disponibilidade, a localização na região Sul apresentou a maior contribuição à situação de segurança alimentar. A probabilidade de um domicílio estar em condições adequadas de consumo alimentar aumenta cerca de 10 p.p. se o domicílio se encontra na região Sul. Para os domicílios urbanos, a probabilidade cai em 3,38 p.p.

Para as variáveis de suficiência, a maior contribuição foi dada pela proporção das despesas com alimentação dentro do domicílio sobre a renda, sendo essa a que apresentou maior efeito marginal entre todas as variáveis analisadas. Assim, para cada aumento de 1 p.p. sobre a parcela de renda gasta com alimentos no domicílio, a probabilidade de o domicílio estar em um nível adequado de ingestão calórica aumenta 69 pontos percentuais. Uma vez que essa variável considera apenas os gastos com alimentos dentro do domicílio, esse efeito pode ser um indicativo de que à medida que mais renda é alocada para a alimentação dentro do domicílio, maior é a probabilidade de o mesmo consumir um nível calórico suficiente.

Sabe-se que domicílios mais pobres tendem a gastar maior proporção de sua renda em alimentos. Claro (2010) mostrou que nesses domicílios o consumo calórico médio é maior em comparação aos domicílios mais ricos, o que pode explicar a relação positiva entre a proporção dos gastos com alimentos e a probabilidade de o domicílio ter uma ingestão calórica suficiente. $\mathrm{O}$ autor ressalta que isso ocorre devido ao fato de que alimentos ricos em calorias tendem a ser relativamente mais baratos em detrimento daqueles ricos em micronutrientes. 


\section{Considerações finais}

A contribuição deste trabalho para a literatura nacional sobre segurança alimentar foi evidenciar uma importante dimensão da mesma, por meio da utilização de uma medida objetiva que a identificasse, possibilitando uma análise sobre o real consumo energético familiar. Dessa forma, foram identificados alguns fatores associados ao consumo calórico suficiente dos domić́lios, como medida do nível de segurança alimentar. Embora apenas $12,4 \%$ dos domicílios ainda não apresentem uma ingestão calórica quantitativamente adequada, os resultados deste trabalho mostram os fatores que contribuem para a incidência de segurança alimentar nesses domicílios, como a situação de pobreza, a carência de itens básicos de infraestrutura e o tamanho do domicílio. Além disso, evidenciou-se o diferencial entre área urbana e rural, onde esta apresentou-se mais favorável à segurança alimentar, devido à importância da produção de subsistência em áreas rurais.

A renda domiciliar per capita, a escolaridade média do domicílio, bem como o número de crianças e idosos determinam o padrão de consumo familiar, influenciando a probabilidade de segurança alimentar de um domicílio. Verifica-se a não existência de diferenças entre o consumo calórico domiciliar suficiente das regiões Nordeste e Sudeste, embora residir na região Sul ainda seja favorável para os domicílios estarem nesse nível de segurança alimentar. Ademais, reforça-se a importância da educação, que contribui para o consumo alimentar.

Pode-se perceber que a probabilidade de segurança alimentar, mesmo em uma situação desfavorável, é alta, correspondendo a cerca de $60 \%$. Nesse contexto, deve-se reconhecer o avanço brasileiro em termos de políticas públicas para o tema nos últimos anos. Cita-se nesse item o Programa Fome Zero", implementado a partir de 2003, e a edição da Lei Orgânica de Segurança Alimentar e Nutricional de 2006, que contribuiu para a construção do arcabouço legal e a definição do Sistema Brasileiro de Segurança Alimentar e Nutricional, que visa assegurar o direito humano à alimentação adequada.

Deve-se ressaltar que o consumo calórico domiciliar, variável de interesse deste trabalho, refere-se apenas à dimensão quantitativa da segurança alimentar. Trata do aspecto prático do problema e ignora a dimensão moral e perceptiva do mesmo. Outras dimensões são igualmente importantes, como a qualidade do alimento disponível e o consumo de nutrientes importantes para uma dieta saudável. Dessa forma, é necessária a investigação da segurança alimentar baseada no consumo de nutrientes importantes para uma alimentação saudável, para que se

(9) O Programa Fome Zero atua por meio de quatro eixos: acesso aos alimentos, fortalecimento da agricultura familiar, geração de renda e articulação, mobilização e controle social. 
possa captar a influência das variáveis analisadas sobre a ingestão qualitativa de alimentos.

\section{Referências bibliográficas}

ANAND, S.; HARRIS, C. J. Food and standard of living: an analysis based on Sri Lankan data. In: DREZE, J. P.; SEN, A. K. (Ed.). The political economy of hunger. Oxford: Clarendon Press, 1990. p. 297-350.

ANVISA. Agência Nacional de Vigilância Sanitária, Resolução - RDC n. 40, de 21 mar. 2001.

BABU, S. C.; SANYAL, P. Food security, poverty and nutrition policy analysis: statistical methods and applications. Oxford: Elsevier, 2009. 340p.

BELIK, W. Perspectivas para segurança alimentar e nutricional no Brasil. Saúde e Sociedade, São Paulo, v. 12, n. 1, p. 12-20, jan./jun. 2003.

BRASIL, Lei n. 11.346, de 15 de setembro de 2006. Cria o Sistema Nacional de Segurança Alimentar e Nutricional - SISAN - com vistas em assegurar o direito humano à alimentação adequada e dá outras providências. Disponível em: http://www.planalto.gov.br/ccivil 03/_Ato2004-2006/2006/Lei/L11346.htm. Acesso em: 20 set. 2011.

CAMELO, R. S.; TAVARES, P. A.; SAIANI, C. C. S. Alimentação, nutrição e saúde em programas de transferência de renda: evidências para o Programa Bolsa Família. Revista Economia, v. 10, n. 4, p. 685-713, 2009.

CLARO, R. M. Influência da renda familiar e dos preços dos alimentos sobre a composição da dieta consumida nos domicílios brasileiros. 123p. Tese (Doutorado em Nutrição em Saúde Pública)- Universidade de São Paulo, São Paulo, 2010.

DUTTA, I.; GUNDERSEN, C. Measures of food insecurity at the household level. In: GUHA-KHASNOBIS, B.; ACHARYA, S. S.; DAVIS, B. (Ed.). Food security: indicators, measurement and the impact of trade openness. Oxford: Oxford University Press, 2007. 396p.

FERREIRA, F. H. G. Os determinantes da desigualdade de renda no Brasil: luta de classes ou heterogeneidade educacional? In: HENRIQUES, R. (Org.). Desigualdade e pobreza no Brasil. Rio de Janeiro: Ipea, 2000. p. 131-158.

FOOD AND AGRICULTURE ORGANIZATION OF THE UNITED NATIONS - FAO. World Food Summit 1996. Disponível em: http://www.fao.org/docrep/003/w3613p/ w3613p00.htm. Acesso em: 10 dez. 2011.

FOOD AND AGRICULTURE ORGANIZATION OF THE UNITED NATIONS - FAO. The state of food insecurity in the world - Addressing food insecurity in protracted crises 2010. Disponível em: http://www.fao.org/docrep/013/i1683e/i1683e.pdf. Acesso em: 17 fev. 2012. 
FOOD AND AGRICULTURE ORGANIZATION OF THE UNITED NATIONS - FAO. The State of Food and Agriculture: women in agriculture - Closing the gender gap for development. Roma, 2011. 160p.

GUBERT, M. B.; BENÍCIO, M. H. D.; SANTOS, L. M. P. dos. Estimativas de insegurança alimentar grave nos municípios brasileiros. Caderno Saúde Pública, Rio de Janeiro, v. 26, n. 8,2010 .

HAUGHTON, J. H.; KHANDKER, S. R. Handbook on poverty and inequality. Washington: The World Bank, 2009. 446p.

HOFFMANN, R. Determinantes da insegurança alimentar no Brasil: análise dos dados da PNAD de 2004. Segurança Alimentar e Nutricional, Campinas, v. 15, n. 1, p. 49-61, 2008.

INSTITUTO BRASILEIRO DE ANÁLISES SOCIAIS E ECONÔMICAS - Ibase. Repercussões do Programa Bolsa Família na segurança alimentar e nutricional: relatório síntese. Rio de Janeiro, 2008. 361p.

INSTITUTO BRASILEIRO DE GEOGRAFIA E ESTATÍSTICA - IBGE. PNAD Segurança Alimentar 2004/2009. Rio de Janeiro, 2010. 183p.

Microdados da POF 2008-2009 (Pesquisa de Orçamentos Familiares). Disponível em: http://www.ibge.gov.br/home/estatistica/populacao/condicaodevida/pof/ 2008 2009/microdados.shtm. Acesso em: 20 abr. 2011.

MALUF, R. S. Segurança alimentar e fome no Brasil: 10 anos da cúpula mundial de alimentação. Ceresan, Relatórios Técnicos, n. 2, 2006.

PAES DE BARROS, R.; HENRIQUES, R.; MENDONÇA, R. Desigualdade e pobreza no Brasil: retrato de uma estabilidade inaceitável. Revista Brasileira de Ciências Sociais, v. 15, n. 42, p. 123-142, 2000.

PÉREZ-ESCAMILLA, R.; SEGALL-CORRÊA, A. M. Food insecurity measurement and indicators. Revista de Nutrição, v. 21, p. 15-26, jul./ago. 2008.

SCHLINDWEIN, M. M.; KASSOUF, A. L. Mudanças no padrão de consumo de alimentos tempo-intensivos e de alimentos poupadores de tempo, por região do Brasil. In: SILVEIRA, F. G. et al. (Org.). Gasto e consumo das famílias brasileiras contemporâneas. Brasília: Ipea, 2007, v. 2, cap. 12, p. 423-462.

SMITH, L. C. The use of household expenditure surveys for the assessment of food insecurity. In: ORGANIZAÇÃO das Nações Unidas para Agricultura e Alimentação FAO. Measurement and assessment of food deprivation and undernutrition. Roma, 2002. $55 \mathrm{p}$.

SOARES, S. S. D. O ritmo de queda na desigualdade no Brasil é adequado? Evidências do contexto histórico e internacional. IPEA, 2008. (Texto para discussão, n. 1.339).

THOMSON, A.; METZ, M. Implications of economic policy for food security: a training manual, 1998. Disponível em: http://www.fao.org/DOCREP/004/X3936E/ X3936E00.HTM. Acesso em: 20 nov. 2011. 
Lorena Vieira Costa / Maria Micheliana da Costa Silva / Marcelo José Braga /Viviani Silva Lírio

TIMMER, C. P.; FALCON, W. P.; PEARSON, S. R. Food policy analysis. Baltimore: The Johns Hopkins University Press, 1983. 301p.

TWEETEN, L. The economics of global food security. Review of Agricultural Economics, v. 21, n. 2, p. 473-488, 1999.

WOOLDRIDGE, J. M. Introdução à econometria: uma abordagem moderna. São Paulo: Cengage Learning, 2008. 